\title{
Pan-Cancer Analysis of the N6-Methyladenosine Eraser FTO as a Potential Prognostic and Immunological Biomarker
}

\author{
Chengwen Zhao',* \\ Yonghui Liu ${ }^{l, *}$ \\ Shaoqing Ju ${ }^{1,2}$ \\ Xudong Wang ${ }^{1,2}$
}

'Department of Laboratory Medicine, Affiliated Hospital of Nantong University, Nantong, Jiangsu, People's Republic of China; ${ }^{2}$ Department of Public Health, Nantong University, Nantong, Jiangsu, People's Republic of China

*These authors contributed equally to this work
Correspondence: Xudong Wang Department of Laboratory Medicine, Affiliated Hospital of Nantong University, 20 Xisi Road, Nantong, Jiangsu, 226006,

People's Republic of China

Tel +86 I395/318000

Email wangxudong88@hotmail.com
Background: Fat mass and obesity-associated protein (FTO) is a critical N6methyladenosine (m6A) demethylase that participates in tumorigenesis and is associated with the prognosis of patients in some cancers. However, the key roles of FTO in pan-cancer are still largely obscure.

Methods: FTO expression levels in pan-cancer were estimated via the Genotype-Tissue Expression (GTEx), Cancer Cell Line Encyclopedia (CCLE), and The Cancer Genome Atlas (TCGA) databases. Univariate survival analysis was used to estimate the effects of FTO on prognosis. In addition, we used the Tumor Immune Evaluation Resource (TIMER) to assess the immune cell infiltration of FTO gene across cancers. The association of FTO expression with immune checkpoint genes expression, DNA mismatch repair (MMR) gene mutation, DNA methyltransferases, microsatellite instability (MSI), and tumor mutational burden (TMB) was investigated using Spearman's correlation analysis. Moreover, Gene Set Enrichment Analysis (GSEA) was utilized to identify critical pathways in cancers. The STRING website was used to reveal the protein-protein interaction (PPI) network of FTO.

Results: FTO was aberrantly expressed across cancers and survival analysis demonstrated that its expression was associated with clinical prognosis of many cancer patients. Specifically, FTO expression was significantly associated with immune infiltrating cells in colon adenocarcinoma, kidney renal clear cell carcinoma, and liver hepatocellular carcinoma. In addition, FTO expression was significantly associated with immune checkpoint genes expression, MMR, DNA methyltransferases levels, TMB, and MSI in multiple cancers. Moreover, the GSEA unveiled that FTO was involved in the regulation of tumors and immune-related signaling pathways. In addition, several m6A related genes were implicated in the PPI network of FTO.

Conclusion: FTO was related to patients' prognosis and tumor immune infiltrates in various cancers, and may serve as a novel and potential prognostic and immune biomarker in human pan-cancer.

Keywords: FTO, biomarker, prognosis, tumor immune, pan-cancer

\section{Introduction}

Fat mass and obesity-associated protein (FTO) is a key N6-methyladenosine (m6A) demethylase located on chromosome 16q12.2. Previous studies have confirmed that FTO regulates the m6A levels of downstream target genes via their 3 '-untranslated regions to influence obesity. ${ }^{1,2}$ Rowing research has suggested that FTO is also closely associated with the occurrence and development of different types of cancers. For instance, FTO may stimulate hepatocellular carcinoma tumorigenesis 
and may be correlated with poor prognosis by mediating the demethylation of PKM2. ${ }^{3}$ Furthermore, the expression of FTO is increased in bladder cancer and promotes tumor growth via the MALAT1/miR-384/MAL2 axis. $^{4}$ In contrast, downregulated expression of FTO is linked to poor survival and to tumor growth and metastasis in lung adenocarcinoma. ${ }^{5}$ Pan-cancer analysis of any gene of interest involves finding commonalities or differences among cancers by integrating cancer expression data of different cancer types based on public databases, ${ }^{6,7}$ and is important for evaluating the clinical prognosis and underlying molecular mechanisms. Although the role of FTO in some cancers has been explored, its key roles in human pan-cancer are still obscure.

The tumor microenvironment (TME) refers to the cellular environment of the tumor. Apart from the tumor cells, the TME also includes various cells such as fibroblasts, immune and inflammatory cells, stromal cells, and endothelial cells. ${ }^{8,9}$ Accumulative evidence has demonstrated that the development and progression of tumors depends on the ongoing coevolution and complex crosstalk with the TME. ${ }^{10,11}$ In particular, as an important element in the TME, immune cells play crucial roles in tumors. For example, the appearance of natural killer (NK) $\mathrm{T}$ cells and CD8+ $\mathrm{T}$ cells in the TME predicted a good clinical outcome in many solid tumors. ${ }^{12,13}$ In contrast, myeloid-derived suppressor cells were a major driver of an immunosuppressive TME and were reported to promote tumor cell invasion and migration in mammary carcinomas. ${ }^{14}$ Regulatory $\mathrm{T}$ cells could also promote the immune escape of tumor cells, and indirectly accelerate the proliferation of tumor cells. ${ }^{15}$ At present, increasing evidence has indicated that targeting the TME, such as via chimeric antigen receptor $\mathrm{T}$ cell therapies and PD-1/PD-L1 checkpoint blockade, could improve treatment outcomes and prognosis for these malignancies. ${ }^{16,17}$ Whereas, immunotherapies are underused in cancers and many patients show poor treatment responses. Consequently, it is an urgent unmet need to identify reliable and effective immunological biomarkers and to explore the relationship between cancers and immunology.

In our study, we comprehensively investigated the relationship between the expression level of FTO and patients' prognosis in 33 types of cancers. Furthermore, we evaluated the correlation between FTO expression and 6 immune infiltrating cells and immune checkpoints genes across cancers. In addition, the correlations of FTO expression with DNA mismatch repair (MMR) genes mutation levels, methyltransferases levels, microsatellite instability (MSI), and tumor mutational burden (TMB) status were analyzed in 33 cancer types. We found that FTO was involved in several cancers and immune-related signaling pathways. These results demonstrated the potential activity of FTO in pan-cancer, indicating a role for FTO as a reliable prognostic and immunological biomarker in some cancers.

\section{Materials and Methods Expression Analysis of FTO and Sample Data Across Cancers}

Different FTO expression in 31 normal tissues (such as liver, lung, and stomach) was assessed via the Genotype-Tissue Expression (GTEx) database (https://gtexportal.org/) and 21 tumor cell lines (such as breast, thyroid, and uterus) information was downloaded from the Cancer Cell Line Encyclopedia (CCLE) database (https://portals.broadinsti tute.org/). In addition, level 3 RNA sequencing data from 33 types of cancers was downloaded for the FTO expression analysis in The Cancer Genome Atlas (TCGA) database (https://portal.gdc.cancer.gov/). We used $\log 2$ conversion to normalize all the expression data. The $\mathrm{R}$ package edgeR was utilized to evaluate FTO expression.

\section{Prognosis Analysis}

The clinical information of all of cancer types was extracted from TCGA database. Univariate survival analysis was utilized to investigate the prognosis of patients in terms of overall survival (OS), disease-free interval (DFI), disease-specific survival (DSS), and progression-free interval (PFI) based on FTO expression levels in 33 types of cancers. Kaplan-Meier curves and forest plots were used for data visualization.

\section{Association Analysis of FTO with the Immune Microenvironment and Immune Checkpoints Genes}

The Tumor Immune Evaluation Resource (TIMER) is a comprehensive database that systematical analyzes immune infiltrates in multiple types of cancers. The Spearman correlation method was utilized to estimate the correlation of FTO expression with tumor immune infiltrating cells $(\mathrm{CD} 4+\mathrm{T}$ cells, $\mathrm{B}$ cells, CD8 $+\mathrm{T}$ cells, macrophages, neutrophils, and dendritic cells) and 47 immune checkpoint genes levels in 33 types of cancers. Furthermore, the 
association analysis of FTO with stromal score across multiple cancers was evaluated via the software Estimate.

\section{Association Analysis of FTO with MMR and DNA Methyltransferases}

The MMR system is a highly conserved biological pathway and plays an important role in sustaining regulating cellular processes and genomic stability. ${ }^{18}$ DNA methylation is an epigenetic mechanism involved in the regulation of gene expression without altering the DNA sequence. Herein, we determined the expression of MMR genes (PMS2, EPCAM, MLH1, MSH2, and MSH6) from TCGA database. Then the relationship between FTO expression and mutation levels of five MMR genes and the expression of four methyltransferases was assessed by Spearman correlation analysis.

\section{Association Analysis of FTO with TMB and MSI}

TMB is defined as the total number of somatic gene coding mutations, such as deletion errors or gene insertions, present in tumor tissue. ${ }^{19}$ MSI refers to a strong mutator phenotype resulting from the loss of DNA mismatch repair activity. ${ }^{20}$ Both TMB and MSI are potential predictive biomarkers for immune checkpoint therapies. We extracted the TMB and MSI data based on TCGA database. The correlations between FTO expression levels and TMB or MSI status were estimated via Spearman's analysis.

\section{Gene Set Enrichment Analysis (GSEA) of FTO in Multiple Cancers}

GSEA is a common analysis tool used to defined whether predefined gene sets show consistent and statistically significant differences between two biological states. ${ }^{21,22} \mathrm{We}$ divided tumor samples into high and low FTO expression groups. The "c2.cp.kegg.v7.1.sym-bols.gmt" was used as the reference gene set for GSEA.

\section{Protein-Protein Interaction (PPI) Network Analysis of FTO in Pan-Cancer}

The STRING website (https://string-db.org/) was utilized to generate the PPI network of FTO with the minimum required interaction score $>0.4$. Cytoscape software (version 3.7.2) was used to visualize the PPI.

\section{Statistical Analysis}

The expression levels of FTO in multiple normal tissues and cancer cell lines were calculated using the Kruskal-
Wallis test. Besides, FTO expression in normal and tumor tissues was analyzed using the Wilcoxon test. Univariate Cox regression analysis was applied to assess the correlation between FTO expression with patients' prognosis. Moreover, the correlation of FTO expression levels with immune checkpoint biomarkers, MMR gene mutation levels, methyltransferases levels, TMB, MSI, and gene expression related to the Wnt signaling pathway was analyzed based on Spearman correlation. A $P$-value $<0.05$ was considered statistically significant in all statistical analyses. In addition, $P<0.05$ and $\mathrm{r}>$ 0.20 was considered significant and positively correlated. In GSEA, $|\mathrm{NES}|>1, P<0.05$, and FDR $<0.25$ were considered statistically significant.

\section{Results}

\section{Expression of FTO Was Altered in Human Pan-Cancer}

First, we used the GTEx and CCLE database to calculate FTO expression in normal tissues and tumor cells (Figure 1A and B). The results showed that FTO exhibited differential expression levels in diverse tissues and cancer cell lines. Next, we evaluated FTO expression in cancer and noncancerous tissues samples from TCGA database (Figure 1C). Given the limited number of normal samples in TCGA database, the GTEx and TCGA databases were combined to investigate differences in FTO expression in 27 tumors. The results showed that FTO was up-regulated in cholangiocarcinoma (CHOL), head and neck squamous cell carcinoma (HNSC), kidney renal clear cell carcinoma (KIRC), acute myeloid leukemia (LAML), brain lower grade glioma (LGG), and pancreatic adenocarcinoma (PAAD), while the expression of FTO was low in the remaining 17 cancers, except for kidney renal papillary cell carcinoma (KIRP), liver hepatocellular carcinoma (LIHC), rectum adenocarcinoma (READ), and stomach adenocarcinoma (STAD) (Figure 1D). In brief, the current results demonstrated that FTO was differentially expressed in a variety of cancers.

\section{Prognostic Potential of FTO in Pan-Cancer}

To explore the relationship between FTO and the clinical prognosis of patients in 33 cancers, datasets from TCGA were used for univariate analysis. The forest plots revealed that FTO significantly influenced the OS, DFI, DSS, and 
A

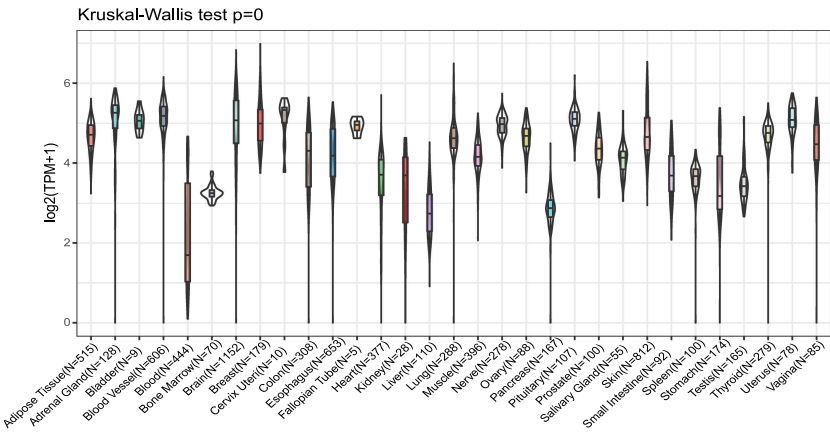

C

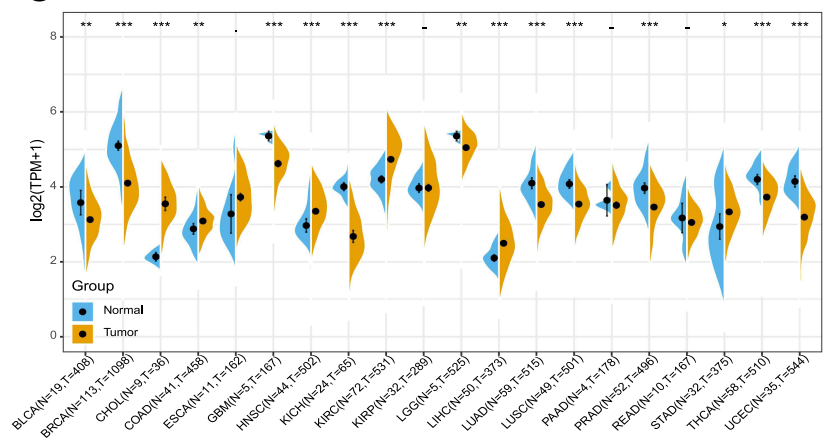

B

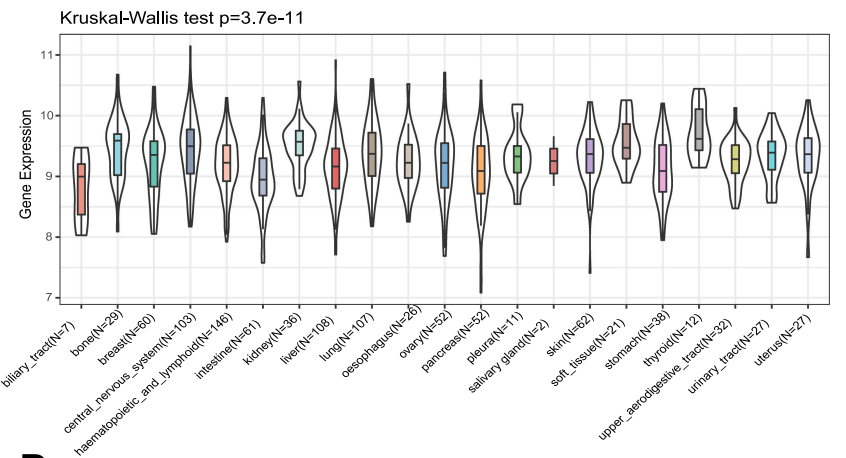

D

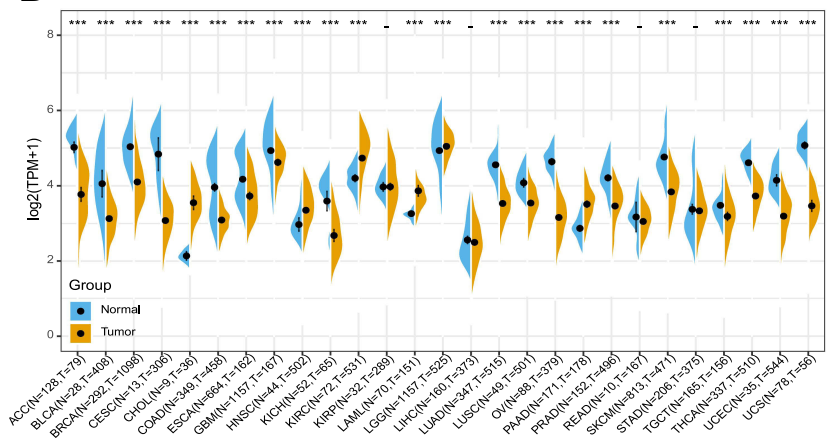

Figure I FTO was abnormally expressed in human pan-cancer. (A) FTO expression levels in 31 normal tissues from the GTEx database. (B) FTO expression levels in 2 I tumor cells from the CCLE database. (C) Differential expression levels of FTO in 20 tumors and paired adjacent noncancerous tissues from TCGA database. (D) FTO expression difference in 27 cancer types integrated from the GTEx database and TCGA database. $(* P<0.05, * * P<0.01$, $* * * P<0.001)$.

PFI of specific tumor types among the 33 cancers evaluated (Figure 2). In particular, the Kaplan-Meier curves revealed that augmented expression of FTO indicated good OS in KIRC $(P<0.0001)$, while high expression of FTO was notably associated with poor OS in bladder urothelial carcinoma (BLCA) $(P=0.00059)$ and STAD $(P=0.0016)$ (Figure 3A-C). Next, we found that augmented levels of FTO unfavorably impacted on DFI in STAD $(P=0.00085)$ (Figure 3D). In addition, we investigated the influence of FTO expression on DSS (Figure 3E-H). High FTO expression was associated with poor prognosis for BLCA $(P=0.00097)$ and STAD $(P<0.0001)$. While the up-regulated FTO was closely associated with favorable prognosis in KIRC $(P<0.0001)$ and thymoma (THYM) $(P<0.0001)$. Furthermore, PFI analysis data revealed that high expression of FTO was an indicator of poor prognosis in BLCA $(P=0.0012)$ and STAD $(P<0.0001)$. In contrast, low expression of FTO was related to poor prognosis for KIRC $(P=0.00018)$ and THYM $(P=0.00061)$ (Figure 3I-L). Overall, the above results implied that the expression of FTO was significantly associated with the prognosis of patients, especially in patients with STAD, BLCA, and KIRC.

\section{FTO Was Associated with Tumor Immune Infiltration and Immune Checkpoint Biomarkers in Multiple}

\section{Cancers}

Immune cell infiltrating tumors are of seminal importance for the development and progression of cancers. ${ }^{23,24}$ Hence, we explored whether FTO expression was associated with the levels of immune infiltration in different cancers. We found that the expression level of FTO was significantly associated with the degree of immune infiltrating cells in a number of human cancer types, and the top three cancers were colon adenocarcinoma (COAD), KIRC, and LIHC (Figure 4A). Furthermore, we used the $\mathrm{R}$ software package Estimate to evaluate the stromal score of each tumor sample. The top three cancers whose FTO expression levels were most significantly positively associated with stromal score were COAD $(\mathrm{R}=0.457, P<$ $0.001)$, PAAD $(\mathrm{R}=0.539, P<0.001)$, and STAD $(\mathrm{R}=$ $0.405, P<0.001$ ) (Figure 4B).

Given that immunotherapies are a crucial treatment approach to reduce and eradicate tumors, the relationship between the expression of FTO and that of 47 immune 
A

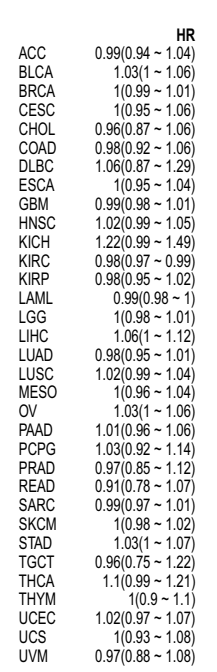

C

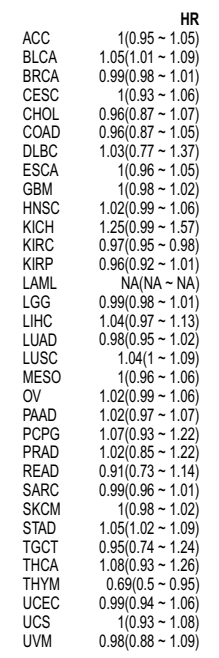

OS

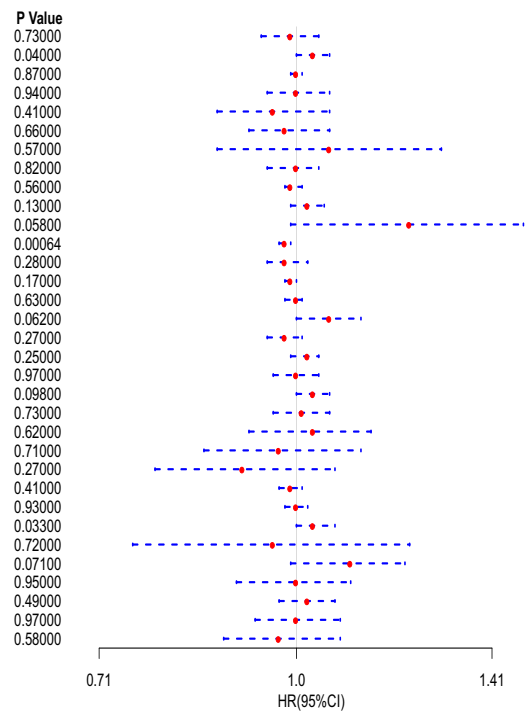

DSS

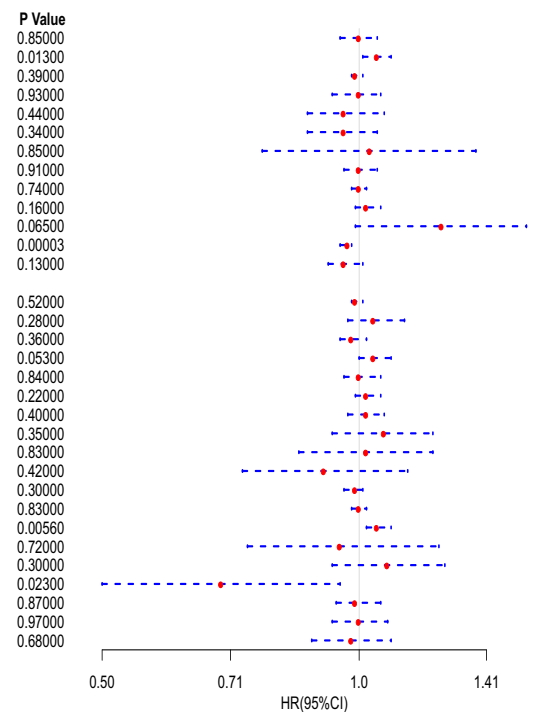

B

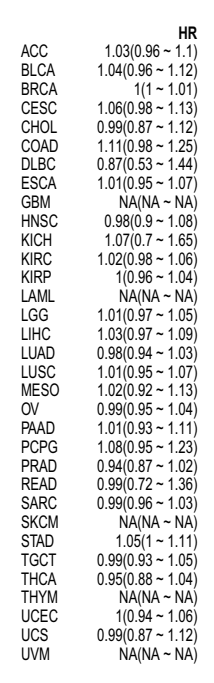

D

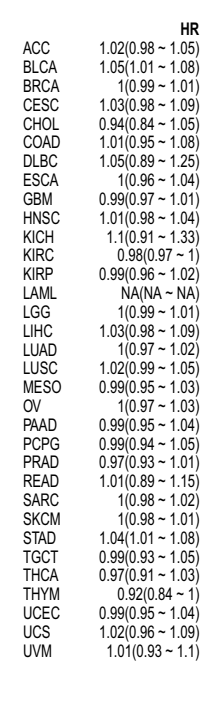

DFI

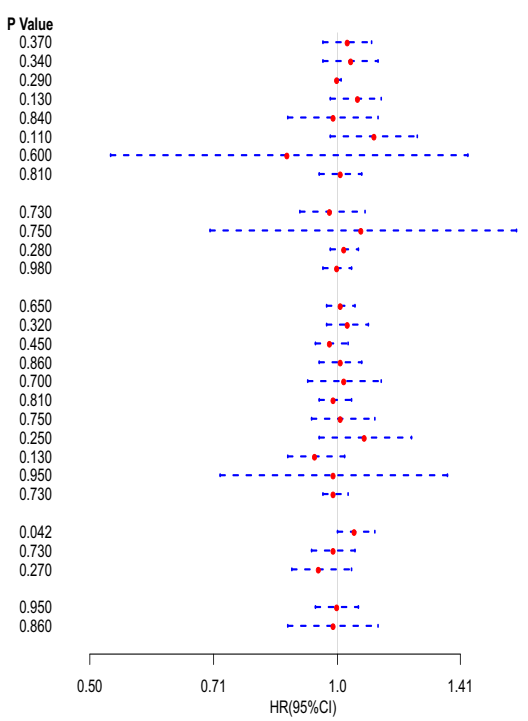

PFI

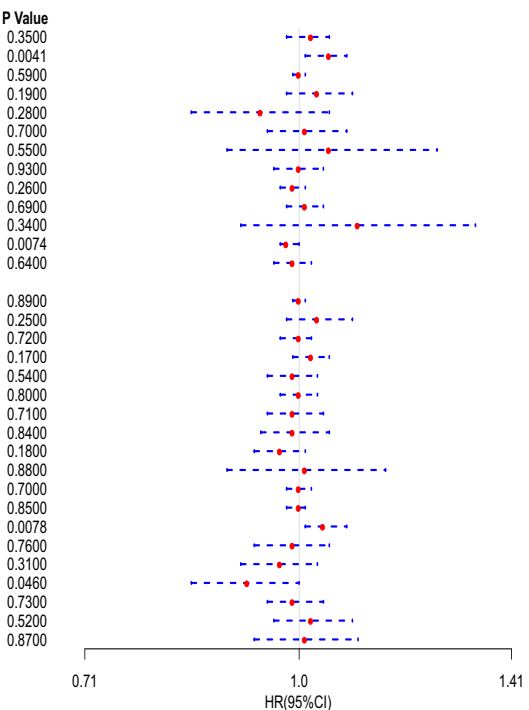

Figure 2 Forest plots of hazard ratios of FTO. (A) The forest plot of the relationship between FTO expression and OS across 33 tumors. (B) The forest plot of the relationship between FTO expression and DFI across 33 tumors. (C) The forest plot of the relationship between FTO expression and DSS across 33 tumors. (D) The forest plot of the relationship between FTO expression and PFI across 33 tumors.

Abbreviations: $\mathrm{HR}$, hazard ratio; $\mathrm{Cl}$, confidence interval.

checkpoint genes was further analyzed. Surprisingly, the analysis indicated that FTO expression was positively associated with the common immune checkpoint genes in multiple cancers, especially in COAD and prostate adenocarcinoma (PRAD) (Figure 4C), indicating that FTO is important for modulating the complex pattern of tumor immune response via the regulation of immune checkpoint genes. Taken together, our study showed that FTO may exert an important role in tumor immunity.

\section{FTO Was Correlated with MMR Gene Expression and DNA Methyltransferases Across Cancers}

The MMR system is composed of a family of enzymes and is responsible for detecting and correcting errors during DNA replication. ${ }^{25}$ Accumulative evidence has demonstrated that alterations in the MMR system can foster tumor progression and targeting MMR can increase the burden of neoantigens, which enhances the 

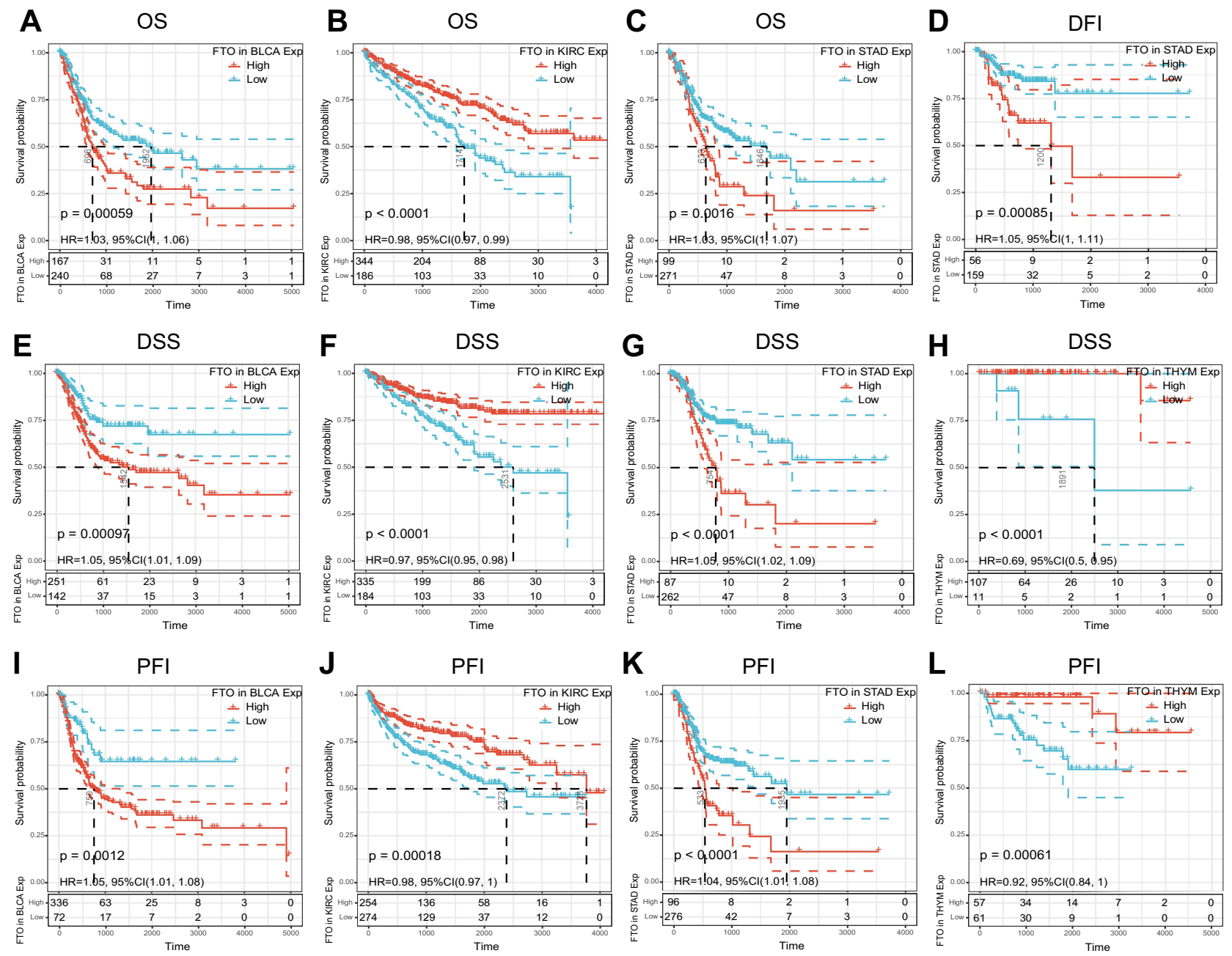

Figure 3 Relationship of FTO expression with patients' prognosis. (A-C) Kaplan-Meier OS curves of FTO expression in BLCA, KIRC, and STAD. (D) The Kaplan-Meier DFI curve of FTO expression in STAD. (E-H) Kaplan-Meier DSS curves of FTO expression in BLCA, KIRC, STAD, and THYM. (I-L) Kaplan-Meier PFI curves of FTO expression in BLCA, KIRC, STAD, and THYM.

response of immune checkpoint inhibitors. ${ }^{26,27}$ To better understand the role of FTO, the association between FTO expression and five MMR genes was evaluated in TCGA datasets. The data revealed that FTO expression was highly correlated with MMR genes across cancers except for LAML (Figure 5A). It is commonly known that hypermethylation within the promoter regions leads to the inactivation of certain tumor suppressor genes and facilitates cancer progression. ${ }^{28}$ As shown in Figure 5B, FTO expression was significantly related to four methyltransferases in all cancers except for LAML. In total, these findings suggested that FTO may regulate the progression of multiple cancers by modulating MMR and DNA methylation.

\section{FTO Affected TMB and MSI in Human Pan-Cancer}

Tumor cells with high TMB have high levels of neoantigens, which are considered to stimulate the anti-tumor response of lymphocytes and to help the immune system recognize tumors. ${ }^{29}$ Our analysis revealed that FTO expression was positively correlated with TMB in LAML, THYM, and uterine corpus endometrial carcinoma (UCEC). Conversely, FTO expression was negatively associated with the TMB in BLCA, breast invasive carcinoma (BRCA), cervical squamous cell carcinoma and endocervical adenocarcinoma (CESC), esophageal carcinoma (ESCA), LIHC, lung adenocarcinoma (LUAD), lung squamous cell carcinoma (LUSC), PRAD, STAD, and 

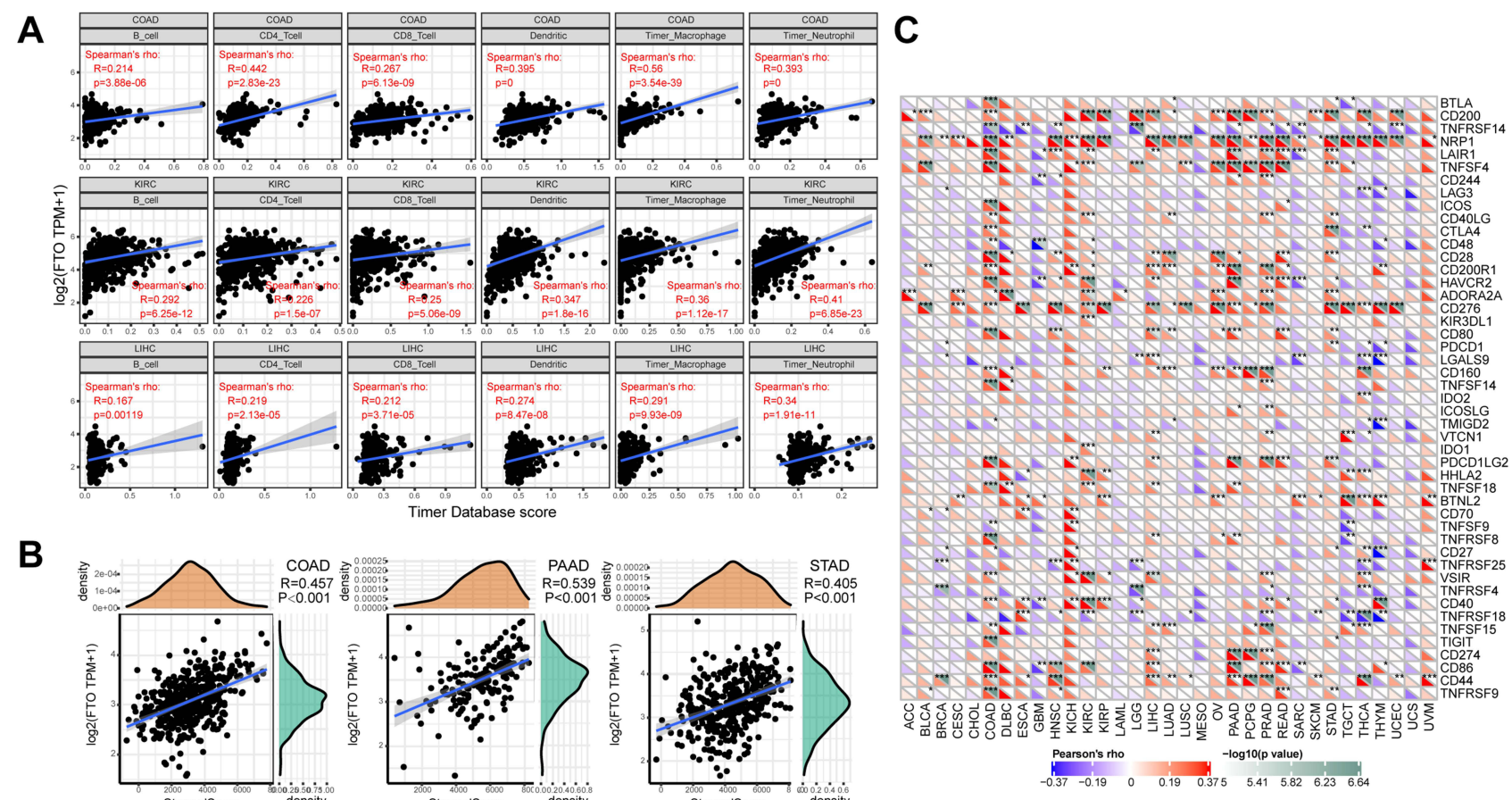

Figure 4 Correlation analysis between FTO expression and immune cell infiltration, stromal score, and immune checkpoint genes across cancers. (A) FTO expression positively correlates with immune infiltrating cells in COAD, KIRC, and LIHC. (B) FTO expression positively correlates with stromal score in COAD, PAAD, and STAD. (C) Correlation analysis between the expression of FTO and 47 immune checkpoint genes across cancers. $(* P<0.05, * * P<0.0 \mathrm{I}$, $* * * P<0.00 \mathrm{I})$.

thyroid carcinoma (THCA) (Figure 6A). Further, MSI has emerged as a key predictor for outcomes of immunotherapy-based strategies. ${ }^{30}$ Herein, we estimated the correlation between FTO expression and MSI using Spearman correlation analysis. The results revealed that FTO expression was positively associated with MSI in testicular germ cell tumors (TGCT), and significantly negatively correlated with MSI in BRCA, lymphoid neoplasm diffuse large B-cell lymphoma (DLBC), HNSC, LUSC, PRAD, skin cutaneous melanoma (SKCM), STAD, and THCA (Figure 6B).

\section{Signaling Pathways and PPI Analysis of FTO Across Cancers}

Stratifying pan-cancer samples according to high or low FTO expression, we observe the potential effects of FTO expression on tumors. Further, GSEA was utilized to analyze the KEGG signaling pathways. The results demonstrated that vascular smooth muscle contraction, Wnt signaling pathways, and endocytosis were the top three enriched pathways in the high expression group (Figure 7A), while the top three signaling pathways, Parkinson's disease, ribosome, and oxidative phosphorylation, were significantly enriched in the low expression group (Figure 7B). Given that abnormal Wnt signaling may disrupt immune surveillance of cancers, leading to resistance to a variety of immunotherapies and to immune evasion, ${ }^{31,32}$ we evaluated the correlation between FTO expression and canonical Wnt signaling pathwayrelated genes, such as CTNNB1, CCND1, CDH2, and c-Myc, in 33 cancers. Specifically, the results indicated that FTO expression was significantly positively correlated with CTNNB1 expression in all cancers (Figure 7C). Furthermore, FTO was positively associated with three other genes in the vast majority of cancers (Figure 7D-F). To further investigate the molecular mechanism involving FTO in tumorigenesis, a PPI network including FTO was constructed (Figure 7G). In particular, m6A related genes, such as METTL3, WTAP, and ALKBH5, were found in this network, indicating that FTO may interact with other m6A genes to drive tumor progression. Collectively, FTO might be involved in multiple pathways and proteins that promote tumor occurrence and progression.

\section{Discussion}

Pan-cancer analysis, which is of seminal importance for understanding differences and similarities among various cancers, can provide new perspectives for cancer 
A

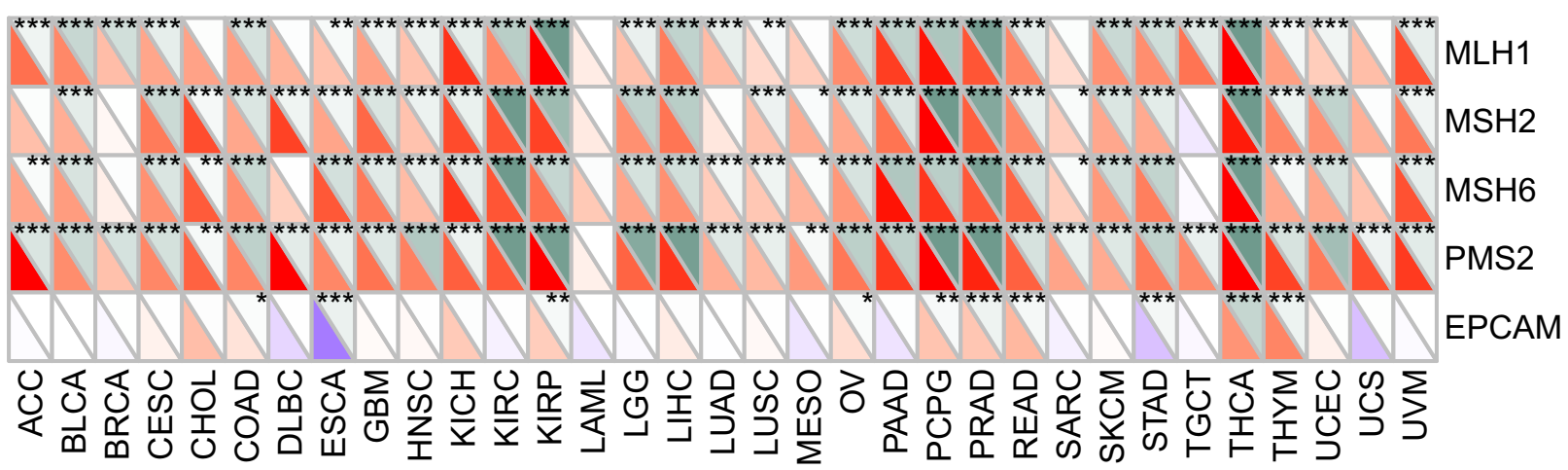

Pearson's rho

$-\log 10$ (p value)

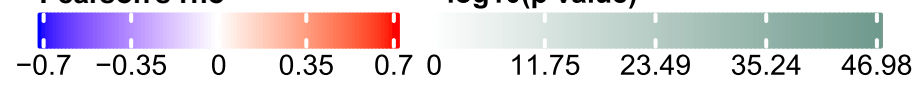

B

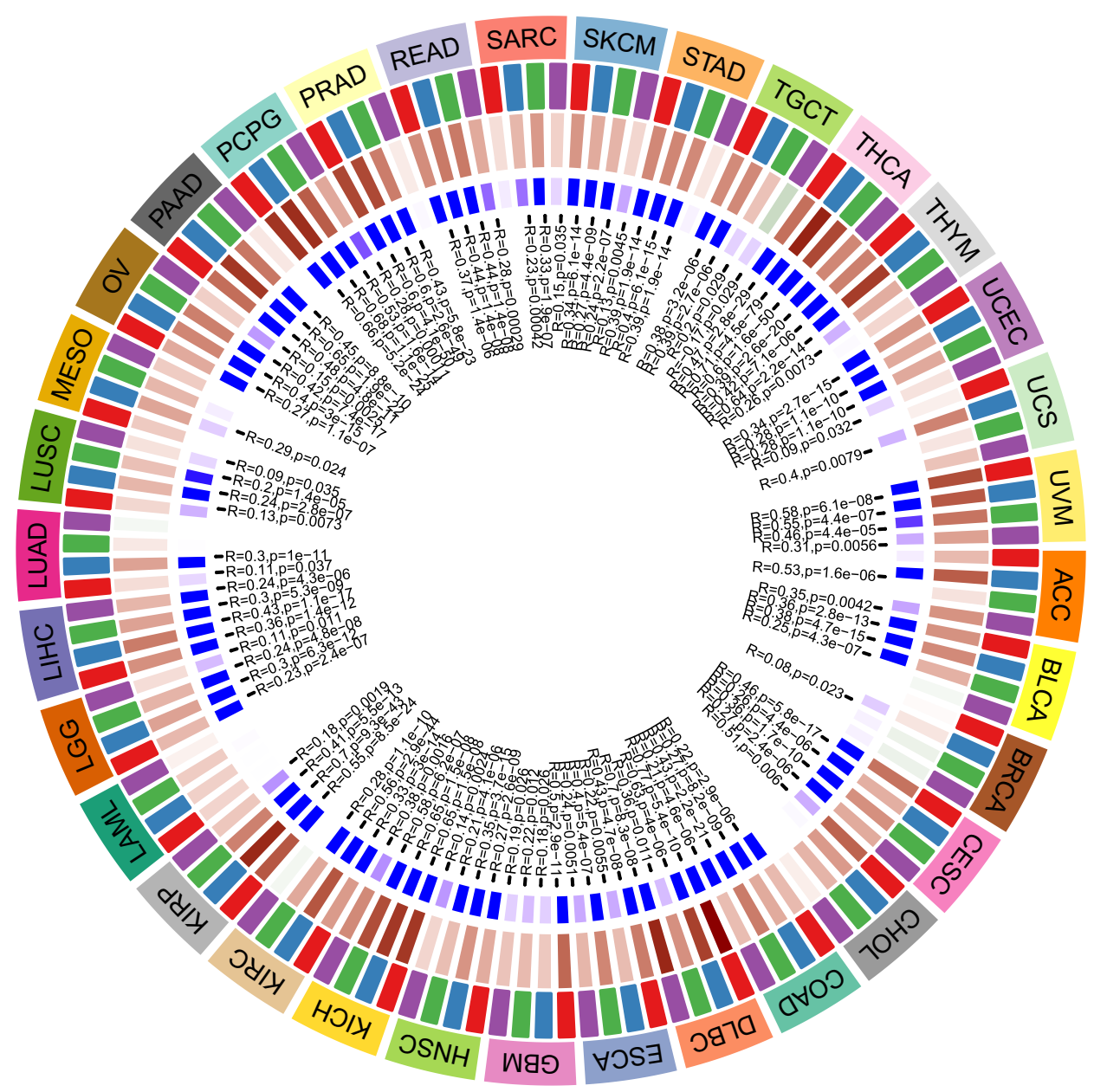

Figure 5 Correlation analysis between FTO expression and MMR gene mutation levels and methyltransferases expression in pan-cancer. (A) Spearman correlation analysis of FTO expression with the expression of five MMR genes in pan-cancer. (B) Spearman correlation analysis of FTO expression with the expression of four methyltransferases in pan-cancer. Red represents DNMTI, blue represents DNMT2, green represents DNMT3A, and purple represents DNMT3B. $(* P<0.05, * * P<0.01$, $* * * P<0.001)$.

prevention and targeted therapy. Increasing studies have described the value of pan-cancer analysis and have revealed the important roles of some driver genes in the development of cancer. ${ }^{33,34}$ FTO, a m6A demethylase, has been identified to as a factor able to regulate the occurrence, progression, and prognosis of some cancers, 

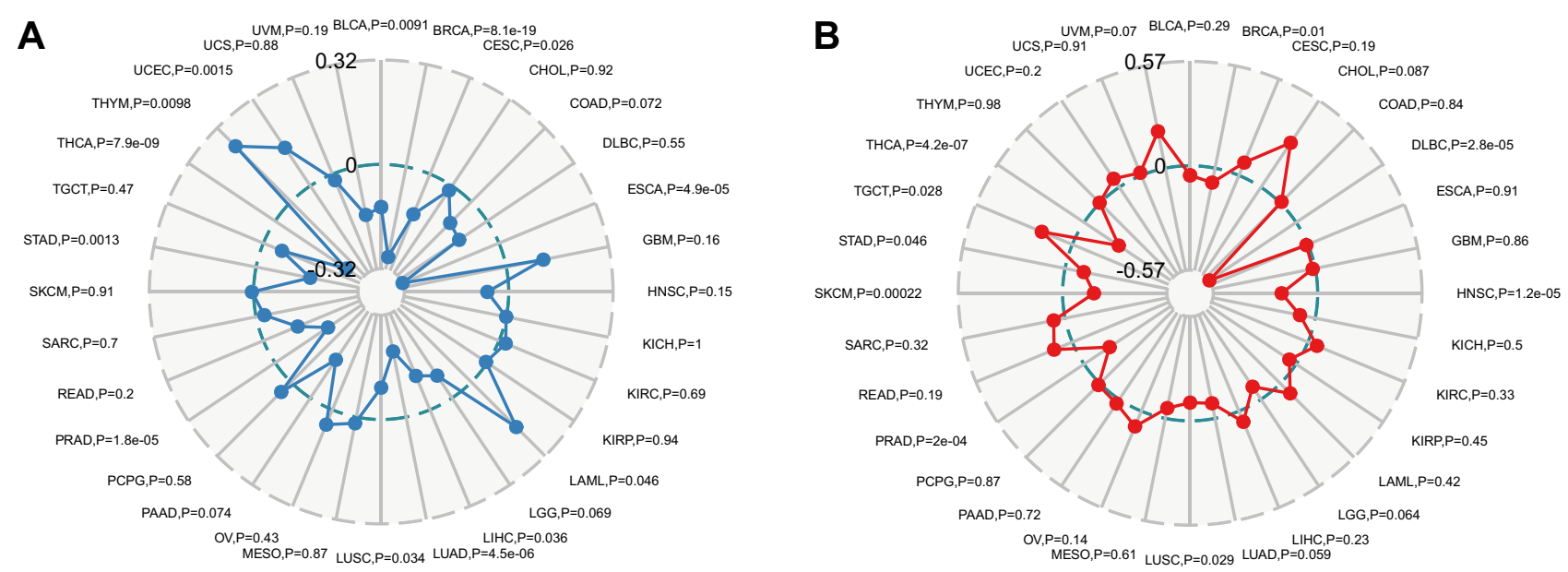

Figure 6 Correlations of FTO expression with TMB and MSI in pan-cancer. (A) The correlation analysis between FTO expression and TMB in pan-cancer. (B) The correlation analysis between FTO expression and MSI in pan-cancer.

including acute myeloid leukemia ${ }^{35}$ and breast cancer. ${ }^{36}$ However, the roles of FTO in cancer development and progression, and whether it can be utilized as a potential biomarker have yet to be investigated. Hence, we first comprehensively elucidated the important role of FTO in human pan-cancer in our study (Supplementary Table 1).

Importantly, FTO was differentially expressed in different cancer types. Univariate survival analysis showed that increased FTO, in particular, predicted poor prognosis of patients in STAD and BLCA. In contrast, up-regulated FTO was positively correlated with prognosis in KIRC and THYM. These results implied that FTO could represent a potential prognostic marker for these cancers.

It is well known that the TME is a major hallmark of cancers. ${ }^{37,38}$ Tumor infiltrating lymphocytes, such as CD4 $+\mathrm{T}$ cells, dendritic cells, and $\mathrm{B}$ cells, are fundamental members of the TME and recognition of their crucial nature in the TME can provide profound insights into tumor immunotherapy. Recent studies have revealed that FTO inhibition may overcome immune evasion by sensitizing leukemia cells to T cell cytotoxicity. ${ }^{39}$ Moreover, $\mathrm{Gu}$ et al found that FTO could promote the polarization of M1 and M2 macrophages. ${ }^{40}$ These results suggested that FTO was involved in tumor immunity in a few types of cancer. However, whether FTO plays a pivotal role in the TME in other cancers is still unknown. Interestingly, our findings revealed that FTO expression was remarkably associated with six immune infiltrating cell types in COAD, KIRC, and LIHC. In addition, we found that FTO expression was strongly correlated with the stromal score in COAD, PAAD, and STAD. Furthermore, the analysis of immune checkpoint markers based on the expression levels of FTO implied that FTO may provide new molecular targets to develop novel immunotherapy modalities. These findings constitute significant progress in identifying the role of FTO in the TME, which strongly suggest that FTO may serve as an immunological biomarker for cancers.

MMR is an evolutionally conserved process and plays an important role in genome stability, DNA replication fidelity, and mutation avoidance, all of which lead to normal biological processes. ${ }^{41}$ However, MMR deficiency leads to an MSI phenotype, which is an indicator of genetic hypermutability. ${ }^{42}$ TMB is a quantitative biomarker that reflects the total number of somatic mutations harbored by tumor cells. Some studies have demonstrated that MMR deficiency, MSI, and TMB are not only prognostic biomarkers for human cancers, but are also predictors of immunotherapy efficacy. For example, the immunotherapy-based anti-PD-1 cancer treatment has been approved for patients whose cancers exhibit MMR deficiency or MSI. ${ }^{43}$ In addition, a high TMB indicates good outcomes of immune checkpoint blockade therapy in diverse cancers. ${ }^{44}$ In the present study, our analysis demonstrated that FTO expression was markedly correlated with MMR gene expression and MSI in different types of cancers. Besides, FTO expression was also correlated with TMB in 13 cancer types. Furthermore, DNA methylation, as the most studied aspect of epigenetics, plays a significant role in the regulation of gene expression, and some studies have confirmed that DNA methyltransferase inhibitors can improve the anti-tumor immune response. ${ }^{45,46}$ Our findings revealed that the expression of 
A

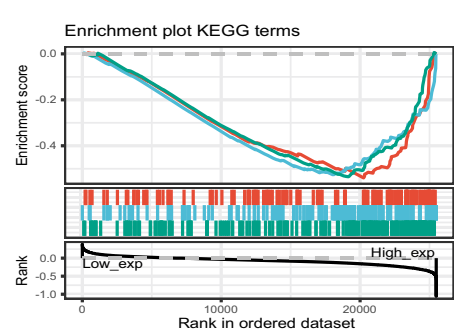

B

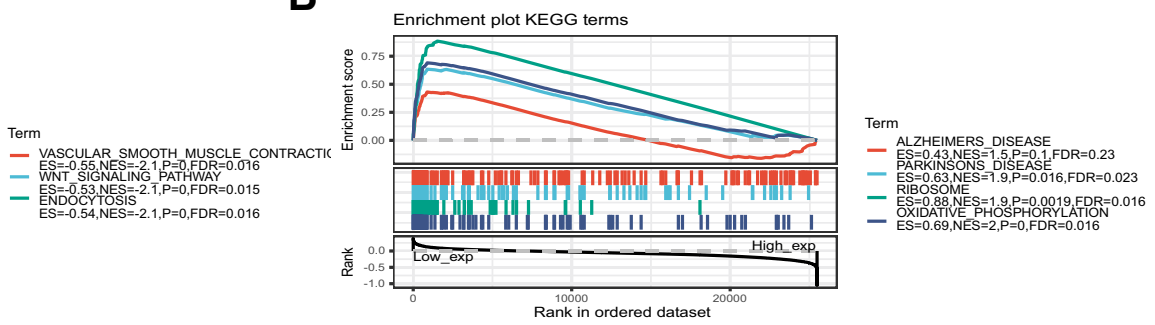

C

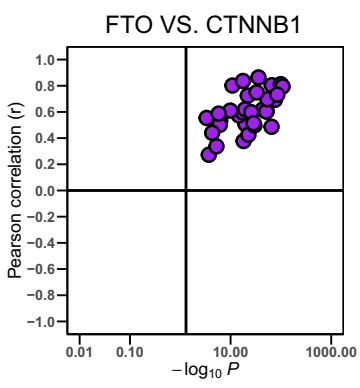

D

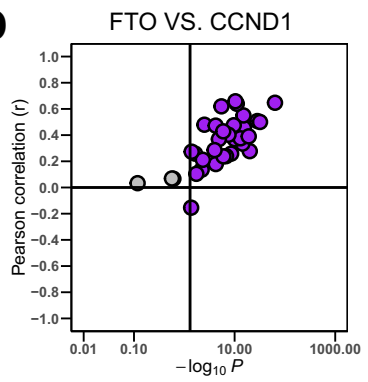

E

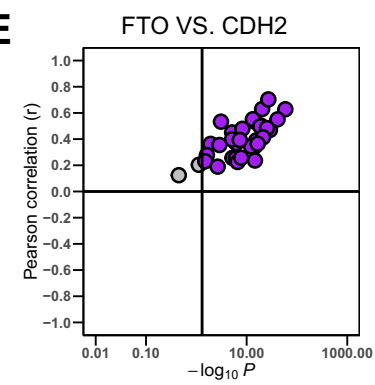

$\mathbf{F}$

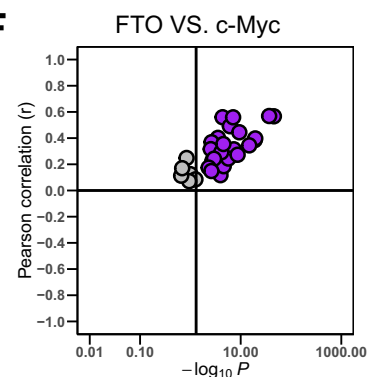

G

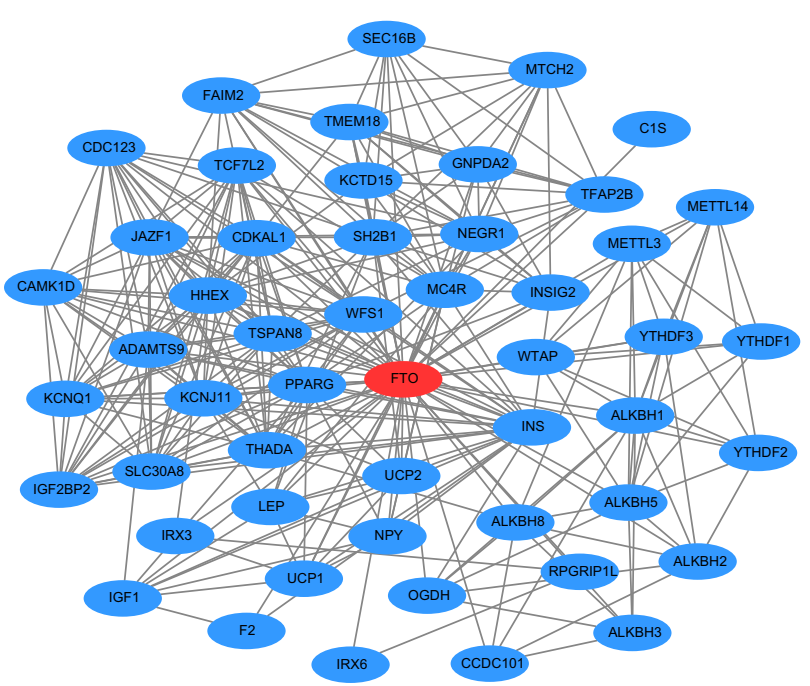

Figure 7 Gene set enrichment, correlation and PPI network analysis of FTO in pan-cancer. (A) The top 3 signaling pathways were enriched in the high FTO expression group in KEGG database. (B) The top 4 signaling pathways were enriched in the low FTO expression group in KEGG database. (C-F) FTO was positively associated with CTNNBI, CCNDI, CDH2, and c-Myc in almost all cancers based on TCGA database. (G) PPI network view from STRING website showed the interaction network of FTO.

FTO was associated with methyltransferase levels in 32 cancers. Hence, aberrant FTO expression, which is highly related to MMR deficiency, MSI, TMB, and DNA methyltransferases, may be a hopeful tumor biomarker used to select patients for the immune treatment. Taken together, compared with reported biomarkers, such as eEF2K, ${ }^{47}$ NTRK $3,{ }^{48}$ and HSPA $7,{ }^{49}$ which predict the prognosis of a single cancer, FTO may serve as a prognostic and immune biomarker for more cancers. However, further experiments are needed to confirm these findings.

Abnormal Wnt signaling pathways play an important part in maintaining homeostasis and are involved in the pathogenesis of many diseases. In particularly, there is mounting evidence that Wnt signaling may regulate immune cell infiltration in the TME. For example, activation of the Wnt signaling pathway is frequently associated with poor spontaneous $\mathrm{T}$ cell infiltration in most cancers. ${ }^{50}$ In addition, Wnt signaling has been implicated in the development of NK cells and NK T cells. ${ }^{51}$ In this study, we not only determined that FTO was significantly enriched in the Wnt signaling pathway, but we also verified the correlation of FTO expression with the expression of key molecules in the Wnt signaling using a comprehensive bioinformatics analysis. Furthermore, a number of m6A-related methylases were identified by analyzing the PPI network of FTO, however, the 
underlying mechanisms remain elusive and warrant further exploration in the future.

\section{Conclusion}

Our study demonstrated that FTO was differentially expressed and associated with patient prognosis and immune infiltrating cells in different cancers. Furthermore, FTO expression was correlated with the immune checkpoint genes, MMR, DNA methylation, MSI, and TMB in numerous cancers. FTO was involved in several pathways that influenced tumorigenesis and tumor immune. PPI analysis indicated the potential impact of m6A-related molecules. Therefore, these findings may provide novel insights into FTO as an effective prognostic and immunological biomarker across cancers. In addition, the study opens new avenues for investigating the potential role of FTO in immunotherapy.

\section{Data Sharing Statement}

The datasets analyzed during the present study are available from TCGA database (https://portal.gdc.cancer.gov/), the GTEx database (https://gtexportal.org/) and the CCLE database (https://portals.broadinstitute.org/). The data used and analyzed in the study is available from the corresponding author on reasonable request.

\section{Funding}

This work was supported by The National Natural Science Foundation of China (No.81972015), the General Project of Jiangsu Provincial Health Commission (H2018074), and the project of Nantong City, and the Science and technology project of Nantong City (JC2019121).

\section{Disclosure}

Dr Xudong Wang reports grants from The National Natural Science Foundation of China, grants from the General Project of Jiangsu Provincial Health Commission, grants from the project of Nantong City, and the Science and technology project of Nantong City, outside the submitted work. The authors report no conflicts of interest in this work.

\section{References}

1. Wu W, Feng J, Jiang D, et al. AMPK regulates lipid accumulation in skeletal muscle cells through FTO-dependent demethylation of $\mathrm{N}^{6}$-methyladenosine. Sci Rep. 2017;7(1):41606. doi:10.1038/srep41606

2. Kang H, Zhang Z, Yu L, Li Y, Liang M, Zhou L. FTO reduces mitochondria and promotes hepatic fat accumulation through RNA demethylation. J Cell Biochem. 2018;119(7):5676-5685. doi: $10.1002 / j c b .26746$
3. Li J, Zhu L, Shi Y, Liu J, Lin L, Chen X. m6A demethylase FTO promotes hepatocellular carcinoma tumorigenesis via mediating PKM2 demethylation. Am J Transl Res. 2019;11(9):6084-6092.

4. Tao L, Mu X, Chen H, et al. FTO modifies the m6A level of MALAT and promotes bladder cancer progression. Clin Transl Med. 2021;11 (2):e310. doi:10.1002/ctm2.310

5. Yang X, Shao F, Guo D, et al. WNT/ $\beta$-catenin-suppressed FTO expression increases $\mathrm{m}^{6} \mathrm{~A}$ of $\mathrm{c}-\mathrm{Myc} \mathrm{mRNA}$ to promote tumor cell glycolysis and tumorigenesis. Cell Death Dis. 2021;12(5):462. doi:10.1038/s41419-021-03739-z

6. Weinstein JN, Collisson EA, Mills GB, et al. The cancer genome atlas pan-cancer analysis project. Nat Genet. 2013;45(10):1113-1120. doi:10.1038/ng.2764

7. Omberg L, Ellrott K, Yuan Y, et al. Enabling transparent and collaborative computational analysis of 12 tumor types within the cancer genome atlas. Nat Genet. 2013;45(10):1121-1126. doi:10.1038/ng.2761

8. Junttila MR, de Sauvage FJ. Influence of tumour micro-environment heterogeneity on therapeutic response. Nature. 2013;501 (7467):346-354. doi:10.1038/nature12626

9. Hinshaw DC, Shevde LA. The tumor microenvironment innately modulates cancer progression. Cancer Res. 2019;79(18):4557-4566. doi:10.1158/0008-5472.CAN-18-3962

10. Wu T, Dai Y. Tumor microenvironment and therapeutic response. Cancer Lett. 2017;387:61-68. doi:10.1016/j.canlet.2016.01.043

11. Huang Q, Hsueh C, Shen Y, et al. Small extracellular vesiclepackaged TGF $\beta 1$ promotes the reprogramming of normal fibroblasts into cancer-associated fibroblasts by regulating fibronectin in head and neck squamous cell carcinoma. Cancer Lett. 2021;517:1-13. doi:10.1016/j.canlet.2021.05.017

12. Simon B, Wiesinger M, März J, et al. The generation of CAR-transfected natural killer $\mathrm{T}$ cells for the immunotherapy of melanoma. Int J Mol Sci. 2018;19(8):2365. doi:10.3390/ijms19082365

13. Jansen CS, Prokhnevska N, Master VA, et al. An intra-tumoral niche maintains and differentiates stem-like CD8 T cells. Nature. 2019;576 (7787):465-470. doi:10.1038/s41586-019-1836-5

14. Yang L, Huang J, Ren X, et al. Abrogation of TGF beta signaling in mammary carcinomas recruits Gr-1+CD11b+ myeloid cells that promote metastasis. Cancer Cell. 2008;13(1):23-35. doi:10.1016/j. ccr.2007.12.004

15. Collison LW, Workman CJ, Kuo TT, et al. The inhibitory cytokine IL-35 contributes to regulatory T-cell function. Nature. 2007;450 (7169):566-569. doi:10.1038/nature06306

16. Kumagai S, Togashi Y, Kamada T, et al. The PD-1 expression balance between effector and regulatory $\mathrm{T}$ cells predicts the clinical efficacy of PD-1 blockade therapies. Nat Immunol. 2020;21 (11):1346-1358. doi:10.1038/s41590-020-0769-3

17. Ma L, Dichwalkar T, Chang JYH, et al. Enhanced CAR-T cell activity against solid tumors by vaccine boosting through the chimeric receptor. Science. 2019;365(6449):162-168. doi:10.1126/science.aav8692

18. Li GM. Mechanisms and functions of DNA mismatch repair. Cell Res. 2008;18(1):85-98. doi:10.1038/cr.2007.115

19. Fancello L, Gandini S, Pelicci PG, Mazzarella L. Tumor mutational burden quantification from targeted gene panels: major advancements and challenges. J Immunother Cancer. 2019;7(1):183. doi:10.1186/ s40425-019-0647-4

20. Boland CR, Goel A. Microsatellite instability in colorectal cancer. Gastroenterology. 2010;138(6):2073-2087. doi:10.1053/j.gastro.200 9.12.064

21. Subramanian A, Tamayo P, Mootha VK, et al. Gene set enrichment analysis: a knowledge-based approach for interpreting genome-wide expression profiles. Proc Natl Acad Sci USA. 2005;102 (43):15545-15550. doi:10.1073/pnas.0506580102

22. Mootha VK, Lindgren CM, Eriksson K-F, et al. PGC-1alpharesponsive genes involved in oxidative phosphorylation are coordinately downregulated in human diabetes. Nat Genet. 2003;34 (3):267-273. doi:10.1038/ng1180 
23. Walsh SR, Simovic B, Chen L, et al. Endogenous $\mathrm{T}$ cells prevent tumor immune escape following adoptive $\mathrm{T}$ cell therapy. $J$ Clin Invest. 2019;129(12):5400-5410. doi:10.1172/JCI126199

24. Fridman WH, Galon J, Dieu-Nosjean M-C, et al. Immune infiltration in human cancer: prognostic significance and disease control. Curr Top Microbiol Immunol. 2011;344:1-24. doi:10.1007/82_2010_46

25. Li Z, Pearlman AH, Hsieh P. DNA mismatch repair and the DNA damage response. DNA Repair. 2016;38:94-101. doi:10.1016/j. dnarep.2015.11.019

26. Germano G, Lamba S, Rospo G, et al. Inactivation of DNA repair triggers neoantigen generation and impairs tumour growth. Nature. 2017;552(7683):116-120. doi:10.1038/nature24673

27. Lord CJ, Ashworth A. The DNA damage response and cancer therapy. Nature. 2012;481(7381):287-294. doi:10.1038/nature 10760

28. Liu T, Qiu X, Zhao X, et al. Hypermethylation of the SPARC promoter and its prognostic value for prostate cancer. Oncol Rep. 2018;39(2):659-666. doi:10.3892/or.2017.6121

29. Jamieson NB, Maker AV. Gene-expression profiling to predict responsiveness to immunotherapy. Cancer Gene Ther. 2017;24 (3):134-140. doi:10.1038/cgt.2016.63

30. Dudley JC, Lin M-T, Le DT, Eshleman JR. Microsatellite instability as a biomarker for PD-1 blockade. Clin Cancer Res. 2016;22 (4):813-820. doi:10.1158/1078-0432.CCR-15-1678

31. Pai SG, Carneiro BA, Mota JM, et al. Wnt/beta-catenin pathway: modulating anticancer immune response. J Hematol Oncol. 2017;10 (1):101. doi:10.1186/s13045-017-0471-6

32. Galluzzi L, Spranger S, Fuchs E, López-Soto A. WNT signaling in cancer immunosurveillance. Trends Cell Biol. 2019;29(1):44-65. doi:10.1016/j.tcb.2018.08.005

33. Hong W, Gu Y, Guan R, Xie D, Zhou H, Yu M. Pan-cancer analysis of the CASP gene family in relation to survival, tumor-infiltrating immune cells and therapeutic targets. Genomics. 2020;112 (6):4304-4315. doi:10.1016/j.ygeno.2020.07.026

34. Zhu L, Wu W, Jiang S, et al. Pan-cancer analysis of the mitophagy-related protein PINK1 as a biomarker for the immunological and prognostic role. Front Oncol. 2020;10:569887. doi:10.3389/ fonc. 2020.569887

35. Li Z, Weng H, Su R, et al. FTO plays an oncogenic role in acute myeloid leukemia as a $\mathrm{N}^{6}$-methyladenosine RNA demethylase. Cancer Cell. 2017;31(1):127-141. doi:10.1016/j.ccell.2016.11.017

36. Xu Y, Ye S, Zhang N, et al. The FTO/miR-181b-3p/ARL5B signaling pathway regulates cell migration and invasion in breast cancer. Cancer Commun. 2020;40(10):484-500. doi:10.1002/cac2.12075

37. Cao R, Yuan L, Ma B, Wang G, Tian Y. Tumour microenvironment (TME) characterization identified prognosis and immunotherapy response in muscle-invasive bladder cancer (MIBC). Cancer Immunol Immunother. 2021;70(1):1-18. doi:10.1007/s00262-02002649-x
38. Di Blasio S, van Wigcheren GF, Becker A, et al. The tumour microenvironment shapes dendritic cell plasticity in a human organotypic melanoma culture. Nat Commun. 2020;11(1):2749. doi:10.1038/ s41467-020-16583-0

39. Su R, Dong L, Li Y, et al. Targeting FTO suppresses cancer stem cell maintenance and immune evasion. Cancer Cell. 2020;38(1):79-96. doi:10.1016/j.ccell.2020.04.017

40. Gu X, Zhang Y, Li D, Cai H, Cai L, Xu Q. N6-methyladenosine demethylase FTO promotes M1 and M2 macrophage activation. Cell Signal. 2020;69:109553. doi:10.1016/j.cellsig.2020.109553

41. Liu D, Keijzers G, Rasmussen LJ. DNA mismatch repair and its many roles in eukaryotic cells. Mutat Res Rev Mutat Res. 2017;773:174-187. doi:10.1016/j.mrrev.2017.07.001

42. Hause RJ, Pritchard CC, Shendure J, Salipante SJ. Classification and characterization of microsatellite instability across 18 cancer types. Nat Med. 2016;22(11):1342-1350. doi:10.1038/nm.4191

43. Hempelmann JA, Lockwood CM, Konnick EQ, et al. Microsatellite instability in prostate cancer by PCR or next-generation sequencing. J Immunother Cancer. 2018;6(1):29. doi:10.1186/s40425-018-0341-y

44. Goodman AM, Kato S, Bazhenova L, et al. Tumor mutational burden as an independent predictor of response to immunotherapy in diverse cancers. Mol Cancer Ther. 2017;16(11):2598-2608. doi:10.1158/ 1535-7163.MCT-17-0386

45. Gallagher SJ, Shklovskaya E, Hersey P. Epigenetic modulation in cancer immunotherapy. Curr Opin Pharmacol. 2017;35:48-56. doi:10.1016/j.coph.2017.05.006

46. Sigalotti L, Fratta E, Coral S, Maio M. Epigenetic drugs as immunomodulators for combination therapies in solid tumors. Pharmacol Ther. 2014;142(3):339-350. doi:10.1016/j.pharmthera.2013.12.015

47. Jiang M, Qi L, Jin $\mathrm{K}$, et al. eEF2K as a novel metastatic and prognostic biomarker in gastric cancer patients. Pathol Res Pract. 2021;225:153568. doi:10.1016/j.prp.2021.153568

48. Zhang Z, Yu Y, Zhang P, et al. Identification of NTRK3 as a potential prognostic biomarker associated with tumor mutation burden and immune infiltration in bladder cancer. BMC Cancer. 2021;21 (1):458. doi:10.1186/s12885-021-08229-1

49. Ding C, He R, Zhang J, Dong Z, Wu J. Pseudogene HSPA7 is a poor prognostic biomarker in Kidney Renal Clear Cell Carcinoma (KIRC) and correlated with immune infiltrates. Cancer Cell Int. 2021;21 (1):435. doi:10.1186/s12935-021-02141-1

50. Li X, Xiang Y, Li F, Yin C, Li B, Ke X. WNT/ $\beta$-Catenin signaling pathway regulating $\mathrm{T}$ cell-inflammation in the tumor microenvironment. Front Immunol. 2019;10:2293. doi:10.3389/fimmu.2019.02293

51. Haseeb M, Pirzada RH, Ain QU, Choi S. Wnt signaling in the regulation of immune cell and cancer therapeutics. Cells. 2019;8 (11):1380. doi:10.3390/cells8111380
International Journal of General Medicine

\section{Publish your work in this journal}

The International Journal of General Medicine is an international, peer-reviewed open-access journal that focuses on general and internal medicine, pathogenesis, epidemiology, diagnosis, monitoring and treatment protocols. The journal is characterized by the rapid reporting of reviews, original research and clinical studies across all disease areas. The manuscript management system is completely online and includes a very quick and fair peer-review system, which is all easy to use. Visit http://www.dovepress.com/ testimonials.php to read real quotes from published authors. 